\title{
Treatment of ptosis by levator resection with adjustable sutures via the anterior approach
}

\author{
H A HYLKEMA AND L KOORNNEEF
}

From the Orbital Centre of the University of Amsterdam, Academic Medical Centre, Meibergdreef 9, 1100 AC Amsterdam, The Netherlands

SUMmARY The authors describe a one-step anterior approach levator resection technique with intraoperative adjustable sutures. Forty-four ptotic eyes were divided into five groups, and the results of this technique were evaluated for each of these five types of ptoses. Our results show that it is not possible to predict the extent of a levator resection preoperatively from the assessment of the levator function and degree of ptosis. We therefore propose this flexible method as the operation of choice for the correction of senile, traumatic and congenital ptosis.

The fact that over 100 techniques for the treatment of ptosis have been reported ${ }^{1-3}$ illustrates that ptosis appears to be difficult to treat, as the postoperative eyelid position is highly unpredictable. We also experienced this in the series of patients presented here. In most cases the anterior approach levator resection technique as described by Beard ${ }^{4}$ was used. According to this method the amount of levator muscle resection is determined before starting the operation and depends on the levator function and the amount of ptosis. In our hands, however, this method caused largely unforeseen postoperative results.

Some surgeons therefore introduced the posterior levator approach ${ }^{5}$ because it opens up the possibility of postoperative adjustment by leaving the sutures in place longer for the cases of undercorrection or removing them earlier if the eyelid is too high postoperatively. We decided to use the anterior approach levator resection technique because it is both easier from the anatomical point of view and leaves the conjunctival surface of the lid (area of accessory lacrimal glands) untouched.

The unpredictable postoperative results formed a strong stimulus to look for a more flexible method to determine the amount of muscle to be resected. In this paper we present our results with an anterior approach levator resection technique with intraoperative adjustable sutures. The amount of levator resection can be determined during the course of the operation, after a provisional fixation of the levator muscle. But to obtain satisfactory results treatment under local anaesthesia is essential.

Correspondence to Dr H A Hylkema.
Before the actual muscle resection is performed the knots are tied and the patient is asked to look straight ahead so that we may evaluate the functional result that can be expected postoperatively. If the position of the eyelid still appears to be too low or too high, the sutures are repositioned in the levator muscle until a good position of the eyelid is achieved. Only then is the actual resection of the levator muscle performed. The patients were selected exclusively on the basis of the amount of ptosis (exceeding $2 \mathrm{~mm}$ ) and levator function (exceeding $3 \mathrm{~mm}$ ). With this modified levator resection technique with adjustable sutures $86 \%$ of the patients with different causes of ptosis had a cosmetically good result after only one surgical procedure.

\section{Material and methods}

The analysis of the amount of ptosis, which is the difference in $\mathrm{mm}$ between the interpalpebral fissures of the eyes and the amount of levator function, was done with a small ruler. The levator function is expressed as the distance in $\mathrm{mm}$ of the superior eyelid between maximal downgaze and upgaze. The frontal muscle should not contribute to this eyelid movement. Its function is checked by pressing the examiner's thumb against the eyebrow while measuring the levator function.

ANTERIOR APPROACH LEVATOR RESECTION TECHNIQUE

A horizontal line is drawn on the eyelid at about $7 \mathrm{~mm}$ from the lid margin to mark the place of the future skin crease. After eversion of the superior 
eyelid $2 \mathrm{ml}$ of lignocaine $2 \%$ with epinephrine is injected into the subconjunctival area. Thus the conjunctiva is separated from Müller's muscle. The upper eyelid is then further anaesthetised subcutaneously with approximately $3 \mathrm{ml}$ of local anaesthetic. A horizontal incision in the skin crease is made through the skin-muscle layer parallel to the muscle fibres on to the tarsal plate. By going upwards in the direction of the upper roof of the orbit the orbital septum is visualised. The septum is opened, the orbital fat is freed, and below the fat the levator muscle is identified. The levator muscle is freed from Müller's muscle. Müller's muscle is not resected. Both the medial and the lateral horns of the levator muscle tendon are cut through. Then a double armed 5.0 catgut suture is positioned in the middle of the levator muscle. The two ends of this suture are provisionally tied to the upper rim of the tarsal plate. The patient is asked to look straight ahead and the position of the operated eyelid can be compared with the other side. The result should be symmetrical. When an under- or overcorrection is still present, this can be overcome by setting the suture higher or lower in the levator muscle or on the tarsal plate. When the result is satisfactory, two more catgut sutures are added $5 \mathrm{~mm}$ medially and laterally from the first one to create an adequate eyelid curve.

\section{Results}

This study was carried out on 41 ptosis patients (38 with unilateral ptosis, 3 with bilateral ptosis) operated on since 1982, when the anterior approach levator resection with adjustable sutures was first introduced. The average age of the patients was 31 years (range 2 to 81 years). Sexes were equally

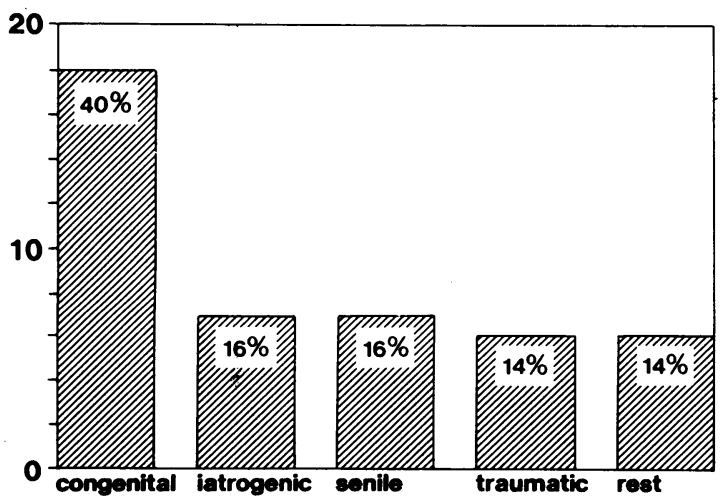

Fig. 1 Causes of ptosis in 44 eyes treated with the anterior approach levator resection. The horizontal axis shows the five subgroups of diagnoses, the abscissa the numbers of eyes in these groups. The percentages are indicated in the bars. distributed in the different groups of diagnoses. Forty-four eyes were treated.

Fig. 1 shows the different causes of ptosis. Fifteen patients had a congenital ptosis, three of them bilaterally, and seven cases were diagnosed as having senile ptosis. Six patients had a traumatic ptosis. Seven patients became ptotic after orbital surgery, while one patient had a ptosis after enucleation. The rest included, among others, cases of Crouzon's disease and oculomotor palsies.

Fig. 2 shows data from 31 out of this group of 44 eyes which were all (44) treated by the anterior approach levator resection with adjustable sutures by one of us (LK) in a uniform and standardised way. The figure shows the levator function of the patients related to the final adjusted amount of resection in $\mathrm{mm}$ of the levator muscle. The area between the lines represents the amount of resection as suggested for the levator resection technique. ${ }^{4}$ In every case the initial amounts of levator resection were those suggested in the literature.

As can be deduced from Fig. 2, it was almost always necessary to correct the amount of resection. There was no clear-cut relationship between the function of the levator muscle and the amount of resection leading to a cosmetically satisfactory result of the ptosis correction. This group consisted of operations on 31 eyes, two patients having a bilateral congenital ptosis. In these bilateral cases the levator functions were $4 / 4$ and $8 / 10 \mathrm{~mm}$ (right/left lid) respectively and the resections needed were $14 / 15$ and $26 / 26 \mathrm{~mm}$. Those operations $(\mathrm{n}=3)$ which did not lead to a satisfactory postoperative result are indicated by an asterisk. Thirteen operations giving another three unsatisfactory postoperative results

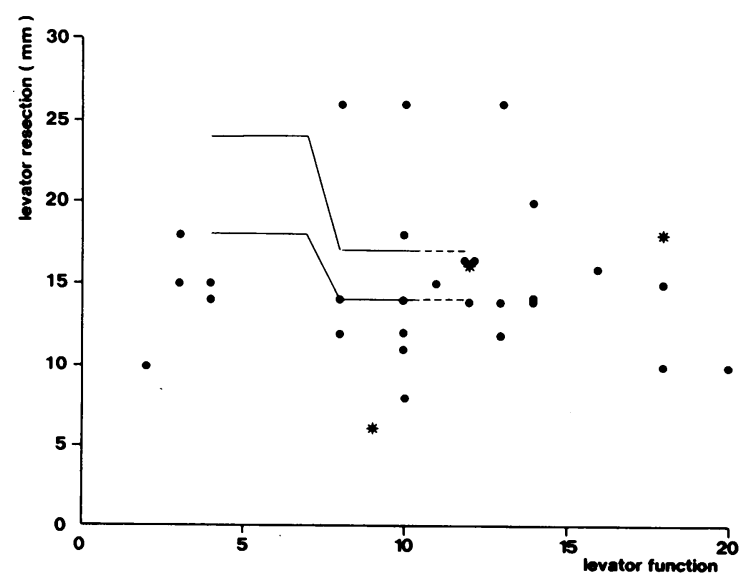

Fig. 2 The preoperative levator function of the ptotic lid related to the actual amount of resection of the levator muscle is shown. The lines represent the upper and lower limits of the amount of resection as proposed by Beard. ${ }^{4}$ 


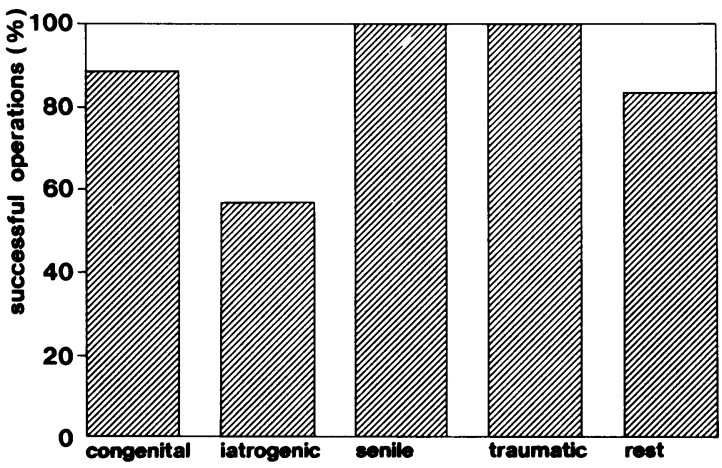

Fig. 3 Percentages of successful operations in the five groups of diagnoses. The horizontal axis shows these five groups, the vertical axis the percentage of successful operations in each group.

are not shown in this figure owing to failure of preoperative documentation.

As can be seen from Fig. 3 there is a marked difference in the percentages of cosmetically successful operations between the five groups of diagnosis. In the groups consisting of senile and traumatic ptoses all patients had a cosmetically satisfactory eyelid position after the levator resection. In the group of congenital ptosis the percentage of successful operations was about $90 \%$. In cases of iatrogenic ptosis only $55 \%$ of the operations led to a good result after one operation. This group was obviously more difficult to treat because we were dealing with ptoses caused by fibrosis and contracted tissues due to surgery and/or radiotherapy. Finally the remaining group contained $80 \%$ of cosmetically good postoperative results. The overall success rate of the whole group after a one-step procedure was $86 \%$.

\section{Discussion}

The results of a one-step anterior approach levator resection technique with intraoperative adjustable sutures are described in this article. This technique was described before by Simonton and Garber. ${ }^{6}$ About $85 \%$ of their patients had a cosmetically satisfying eyelid position postoperatively. Making use of a nearly identical technique, they operated on 44 eyes, and of these four eyelids required a revision of the procedure. These authors, however, do not mention data about diagnoses, the amount of resection done, and the pre- and postoperative measurements of their patients. Collin and Tyers ${ }^{7}$ treated 55 eyes with ptosis due to an aponeurotic defect, ranging from 1 to $5 \mathrm{~mm}$, making use of a posterior approach levator resection with adjustable sutures. ${ }^{5}$ Ten of the 55 eyes $(18 \%)$ needed a second approach by removal of sutures or stretching of the eyelid. Eventually $81 \%$ of the patients showed functional and cosmetically good results.

In our heterogeneous group (in which some of the patients are more difficult to treat than others), treated by an intraoperative monitoring surgical technique, only $14 \%$ of the patients had an unsatisfactory eyelid position postoperatively. This relatively good result can be explained by the fact that the anterior approach levator resection with adjustable sutures is a flexible method yielding good results even in difficult conditions. These results support the assumption that in ptosis a number of factors, such as anatomical, ${ }^{8}$ neurological, and muscular variations, ${ }^{39}$ lead to an individual eyelid position. The balance between the strength of the levator muscle and the orbicular muscle may play a major part in the positioning of the eyelid. These hypotheses, however, need further analysis.

The results of this study and data from the literature show that the results of ptosis operations are characterised by unpredictability and the need for individual assessment, not solely by the amount of ptosis and levator function as determined preoperatively.

\section{References}

1 Jones LT. The anatomy of the upper eyelid and its relation to ptosis surgery. Am J Ophthalmol 1964; 57: 943-53.

2 Jones LT, Quickert MH, Wobig LJ. The cure of ptosis by aponeurotic repair. Arch Ophthalmol 1975; 93: 629-34.

3 Mustarde JC. Correction of ptosis by split level lid resection. Clin Plast Surg 1978; 5: 533-5.

4 Beard C. Ptosis. St Louis: Mosby, 1981.

5 Collin JRO. A manual of systemic eyelid surgery. New York: Churchill Livingstone, 1983.

6 Simonton JT, Garber PF. Monitored ptosis surgery. Adv Ophthalmic Plast Reconstruct Surg 1982; 1: 167-81.

7 Collin JRO, Tyers AG. Posterior approach repair of aponeurotic defects-review of 55 cases. Adv Ophthalmic Plast Reconstruct Surg 1982; 1: 135-43.

8 Collin JRO, Beard C, Wood I. Experimental and clinical data on the insertion of the levator palpebrae superioris muscle. Am J Ophthalmol 1978; 85: 792-801.

9 Koornneef L. Eyelid and orbital fascial attachments and their clinical significance. Eye, in press.

Accepted for publication 21 April 1988. 